\title{
Proper orthogonal decomposition preprocessing of infrared images to rapidly assess stress-induced heat source fields
}

\author{
Adil Benaarbia, André Chrysochoos \\ Mechanics and Civil Engineering Laboratory, University of Montpellier, Montpellier, France
}

\begin{abstract}
An image processing technique using the proper orthogonal decomposition (POD) of infrared thermal data was developed to improve the speed of assessment of 2D heat source fields accompanying mechanical transformation. This method involved the generation of a reduced orthonormal basis to approximate thermal fields prior to heat source estimation. The robustness of the method was first assessed using a penalising benchmark test. This test involved artificially setting several tricky situations that arise in practice (high diffusivity, low signal-to-noise ratio, complex heat source distribution, etc.). Application of the method to several experimental temperature fields obtained by an infrared focal plane array camera is then presented. The error between the POD approximated solution in terms of heat sources and a reference solution, computed via a local least squares fitting method, was found to be negligible, thus confirming the efficiency and advantages of the POD preprocessing technique - the method enabled us to obtain a reliable estimate of heat sources while drastically reducing the computation cost in terms of CPU time.
\end{abstract}

\section{KEYWORDS}

Quantitative infrared techniques; proper orthogonal decomposition; heat source estimate; dissipation fields

\section{Introduction}

Different types of heat source accompany material deformation. First, the so-called dissipative source is induced by irreversible deformation mechanisms such as plasticity, viscosity and damage. The second type is related to strong interactions that may occur between mechanical, thermal and microstructural states of the material. This is the so-called thermomechanical coupling heat source induced by thermal dilatation, first-order phase change, rubber elasticity, etc. Strain-induced heat sources usually generate a temperature variation, which is consequently chosen as a state variable in thermomechanical modelling of the material behaviour [1]. However, this temperature variation is not exclusively related to the material deformation mechanisms insofar as it is affected by the heat source distribution, heat conduction transfers within the matter, and heat exchanges between the tested specimen and the surroundings. Conversely, heat sources are directly connected to the constitutive laws of the material behaviour, with coupling sources being related to state laws, while dissipative sources reflect the irreversibility associated with kinetic laws [2,3]. These 
calorimetric data are essential for anyone wanting to develop behavioural models using thermomechanical formalism.

Over the last 20 years, with the incredible advances that have been achieved in quantitative infrared techniques, numerous specialised experimental data processing techniques have been developed to get increasingly reliable heat source assessments [4-8]. Among these techniques, many are based on direct estimate of the heat source distribution using the local expression of the heat diffusion equation and thermal data provided by an IR camera. However, as temperature fields can only be monitored at the specimen surface, thin flat specimens have generally been used for 2D approximation of the heat diffusion problem. The heat sources are then estimated via direct estimate of the partial derivative operators present in the local form of the heat diffusion equation. Indeed, by averaging the 3D heat diffusion equation [4] over the sample thickness, a 2D thermal diffusion model can be obtained as follows:

$$
\rho C\left(\frac{\partial \bar{\theta}}{\partial t}+\boldsymbol{v} \cdot \nabla \bar{\theta}+\frac{\bar{\theta}}{\tau_{\text {th }}^{2 \mathrm{D}}}\right)-k_{c}\left(\frac{\partial^{2} \bar{\theta}}{\partial x^{2}}+\frac{\partial^{2} \bar{\theta}}{\partial y^{2}}\right)=\bar{s}^{h},
$$

where $\rho, C$ and $k_{c}$ represent the mass density, specific heat and thermal conductivity, respectively. For simplicity, these material parameters are assumed to be physical constants and the velocity field $v$ independent of the depth-wise coordinate. The terms $\bar{\theta}$ and $\bar{s}^{h}$ in turn stand for the temperature variations and heat sources averaged over the sample thickness. Temperature variations are assessed with respect to a room temperature $T_{0}$ that is taken to be spatially uniform and temporarily constant. The $\tau_{\mathrm{th}}^{2 \mathrm{D}}$ parameter represents the time constant characterising perpendicular heat loss to the specimen surface. This constant depends on the thermophysical properties of the material under investigation, the geometry of the sample and the heat transfer coefficient $h$, while assuming that heat exchanges by conduction, radiation and convection between the sample and the surrounding air can be correctly approximated by a linear function of the thermal disequilibrium [4]. This time constant can then be formulated as:

$$
\tau_{\mathrm{th}}^{2 \mathrm{D}}=\frac{\rho C e}{2 h}
$$

where e stands for the sample thickness. Note that the convective terms in Equation (1), corresponding to the scalar product of the displacement velocity $\boldsymbol{v}$ and temperature gradients $\nabla \bar{\theta}$ in the total time derivative of the temperature, are often assumed to be negligible. Its estimate is, however, possible if another quantitative imaging technique is used to get the displacement and velocity fields. In reference [9], digital image correlation and infrared thermography (IRT) techniques were combined to assess these convective terms. In some cases, they cannot be neglected (e.g. necking lips of polymers [10], narrow strain localisation zones [11]). We nevertheless ignored them since we intended to focus on the efficiency of proper orthogonal decomposition (POD) techniques in rapidly filtering thermal images before heat source computation. Note, however, that generalisation of the method should not be problematic. POD preprocessing could also be applied to kinematic full-field measurements.

In fact, one of the main difficulties associated with heat source assessment concerns the thermal data, which are discrete and noisy by nature. This drastically complicates estimation of the partial derivative terms in the heat diffusion equation. The signal-to-noise ratio and 
spatiotemporal sampling rates are often not high enough to compute relevant heat source distributions. In particular, the 2D Laplacian operator is a heavy noise amplifier.

It is therefore essential to have specific filtering or fitting techniques able to compute such derivative terms. Several processing techniques have been described in the literature: (i) projection of thermosignals onto eigenfunctions associated with the spatial differential operator of the heat equation [12], Fourier techniques involving periodic expansion of images and convolutive filtering [4], and local least-squares approximations [8]. Most of these techniques were used to investigate thermomechanical phenomena such as strain localisation in steels and polymers [4,13,14], thermoelastic effects accompanying deformation, [1] phase transformation in shape memory alloys [15-19], dissipated and stored energy in elastoplastic materials [20,21], etc.

POD was recently applied to process thermoprofiles in order to assess 1D heat source distributions in an ultrasonic cycle fatigue framework [22]. POD preprocessing was compared to previous techniques (convolutive filtering, local least-squares fitting, use of analytical eigenfunctions). A comparative study highlighted the promising features of POD techniques not only in terms of heat source assessment efficiency but also of data processing speed.

The current study deals with the use of POD to estimate 2D heat source fields involved during mechanical tests. The first part of the study consists of theoretical validation of the POD technique. The influence of thermal noise on the heat assessment, which is a key issue here, is widely discussed. The second part of the study presents an application of POD to several experimental temperature fields obtained by IRT during a fatigue test on a composite material. The experimental identification of fatigue lifespan criteria is particularly time consuming and a great effort is currently being made to develop new rapid methods for characterisation of fatigue strength properties of materials and structures [23].

\section{Fundamentals of POD}

POD techniques have been widely used in model order reduction, which aims to lower the computational complexity of large-sized problems, e.g. in simulation of large-scale dynamical systems and control systems. By a reduction of the model's associated state space dimension or degrees of freedom, an approximation of the original model is computed. Numerous examples have been provided in many image processing domains [24], signal analysis and data compression [25], turbulence models and coherent structures in fluids [26,27], modelling and control of chemical reaction systems [28-31], etc.

The principle of POD techniques consists of taking a given collection of experimental (let us say thermal) data and creating an orthonormal basis constituted by functions estimated as solutions of an integral eigenvalue Fredholm problem [32-38]. These eigenfunctions are, by definition, characteristics of the most probable outcomes of experimental thermal data. In practice, it has been pointed out that the noise reduction obtained by truncating the POD solution was better than convolutive filtering and/or local least-squares fitting [22,39]. For the POD technique, the CPU times necessary to project the thermal data were almost negligible compared to those of other preprocessing techniques.

Simply from a mathematical standpoint, POD techniques allow us to consider a scalar function $\vartheta(x, y, t)$ (hereafter depicting the time course of the 2D temperature field) as a finite sum in the separated-variables form: 


$$
\vartheta(x, y, t) \simeq \sum_{p=1}^{p} a_{p}(t) \Phi_{p}(x, y)
$$

where $(x, y)$ can be viewed as spatial coordinates, $t$ as a temporal coordinate, $a_{p}$ as of POD approximation coefficients and $\left(\Phi_{p^{\prime}} p=1, \ldots P\right)$ as a set of orthogonal functions subsequently called proper orthogonal modes (POMs). Equation (3) indicates that the approximation converges towards the $\vartheta$ fields when $P$ approaches infinity. From a more physical standpoint, the POD technique thus involves extracting the most energetic modes that capture most of the energy of $\vartheta$ fields. This implicitly supposes that the thermal noise signal energy remains low compared to the thermosignal induced by the material transformation. The calculation of POD approximation coefficients $a_{p}$ closely depends on the nature of the basis functions $\Phi_{p}$. These POMs are only spatial functions and can be used to approximate $\vartheta$ fields when combined with the set of coefficient $a_{p}$. So, for a given orthonormal set of basis functions $\Phi_{p}$

$$
\left\langle\Phi_{p}(x, y), \Phi_{q}(x, y)\right\rangle=\delta_{p q}=\left\{\begin{array}{ll}
1 & p=q \\
0 & p \neq q
\end{array},\right.
$$

where $\delta_{p q}$ is Kronecker's delta symbol and the notation $\left\langle\Phi_{p^{\prime}} \Phi_{q}\right\rangle=\iint \Phi_{p} \Phi_{q} \mathrm{~d} x \mathrm{~d}$ y gives the inner product, while the coefficients $a_{p}$ can be obtained from

$$
a_{p}(t)=\left\langle\vartheta(x, y, t), \Phi_{p}(x, y)\right\rangle
$$

For selecting the function $\Phi_{p^{\prime}}$ it is convenient to use the orthonormality condition as $a_{p}$ depends only on $\Phi_{p}$ and not on the other $\Phi_{q}, q \neq p$.

Assume now that $\vartheta$ fields can be experimentally available on a spatial grid of size $\alpha_{x} \times \alpha_{y} \times \alpha_{t^{\prime}}$ i.e. with $a_{x}$ gridpoints in the $x$ direction, $a_{y}$ gridpoints in the $y$ direction and $a_{t}$ gridpoints in time. Let us also introduce the Euclidean space $\mathbb{R}^{\alpha_{x} \times \alpha_{y}}$. The reduced basis representation (see Equation (3)) can thus be equivalent to finding the orthonormal basis function $\left\{\Phi_{p}\right\}_{p=1, \ldots, P}$ solutions of the following minimisation problem

$$
\min _{\Phi_{p}} \sum_{i, j, \kappa=1}^{\alpha_{x}, \alpha_{y}, \alpha_{t}}\left\|\vartheta\left(x_{i}, y_{j}, t_{\kappa}\right)-\sum_{p=1}^{P}\left\langle\vartheta\left(x_{i}, y_{j}, t_{\kappa}\right), \Phi_{p}\left(x_{i}, y_{j}\right)\right\rangle_{\mathbb{R}^{\alpha_{x} \times \alpha_{y}}} \Phi_{p}\left(x_{i}, y_{j}\right)\right\|_{\mathbb{R}^{\alpha_{x} \times \alpha_{y}}}^{2}
$$

where $\|\cdot\|_{\mathbb{R}^{a_{x} \times \alpha_{y}}}=\sqrt{\langle. . .\rangle_{\mathbb{R}^{\alpha_{x} \times \alpha_{y}}}}$ defines the norm associated with the canonical inner product in $\mathbb{R}^{\alpha_{x} \times \alpha_{y}}$, and $i=1,2, . ., \alpha_{x^{\prime}} j=1,2, . ., \alpha_{y^{\prime}} k=1,2, . ., \alpha_{t}[40,41]$.

A practical way to solve the minimisation problem (Equation (6)) is to arrange the data-set in a matrix $\Theta \in \mathbb{R}^{\alpha_{z} \times \alpha_{t}}$, with $\alpha_{z}=\alpha_{x} \times \alpha_{y}$ the so-called snapshot data matrix. The data arrangement was used here to reduce the size of the tridimensional array $\vartheta$ into a bi-dimensional array $\Theta$. The solutions of the minimisation problem (i.e. $\left\{\Phi_{p}\right\}_{p=1, \ldots, p}$ ) are thus given by the truncated singular value decomposition (SVD) of length $\alpha_{z}$ of the matrix $\Theta$. The SVD guarantees the existence of real numbers $\varrho_{1} \geq \varrho_{2} \geq \ldots \geq \varrho_{r}>0$, where $r=\min \left(\alpha_{z}, \alpha_{t}\right)$ and orthogonal matrices $R \in \mathbb{R}^{\alpha_{z} \times \alpha_{z}}$ and $S \in \mathbb{R}^{\alpha_{t} \times \alpha_{t}}$, such that

$$
R^{T} \Theta S=\left(\begin{array}{cc}
L & 0 \\
0 & 0
\end{array}\right)=\Sigma \in \mathbb{R}^{\alpha_{z} \times \alpha_{t}}, \quad L=\operatorname{diag}\left(\varrho_{1}, \varrho_{2}, \ldots, \varrho_{r}\right) \in \mathbb{R}^{r \times r},
$$


where (.) ${ }^{T}$ stands for the transpose of the matrix (.). The zeros in Equation (7) denote matrices of appropriate dimensions. Moreover, the vectors $\left\{R_{i}\right\}_{i=1}^{r}$ and $\left\{S_{i}\right\}_{i=1}^{r}$ satisfy

$$
\Theta S_{i}=\varrho_{i} R_{i} \text { and } \Theta^{T} R_{i}=\varrho_{i} S_{i}, \quad \text { for } i=1, \ldots, r \text {. }
$$

These are the eigenvectors of $C^{\text {right }}=\Theta \Theta^{T}$ and $C^{\text {left }}=\Theta^{T} \Theta$ respectively associated with the eigenvalues $\omega_{i}=\rho_{i}^{2}>0, i=1, \ldots, r$. Matrix $C^{\text {right }}$ is the so-called correlation matrix. By construction, this matrix is symmetrical, positive and semidefinite.

From Equations (7) and (8), we can deduce that

$$
\Theta=R \Sigma S^{T} .
$$

It follows that $\Theta$ can be expressed as

$$
\Theta=R^{r} L\left(S^{r}\right)^{T},
$$

where the matrices $R^{r} \in \mathbb{R}^{\alpha_{z} \times r}$ and $S^{r} \in \mathbb{R}^{\alpha_{t} \times r}$ are given by

$$
R_{i j}^{r}=R_{i j}, \quad S_{k j}^{r}=S_{k j}
$$

for $i \in\left[1, \ldots, \alpha_{z}\right], j \in[1, \ldots, r]$ and $k \in\left[1, \ldots, \alpha_{t}\right]$.

Now, noting that $M^{r}=L\left(S^{r}\right)^{T} \in \mathbb{R}^{r \times \alpha_{t}}$, we can formulate Equation (10) as

$$
\Theta=R^{r} M^{r} \text {. }
$$

Thus, the column space of $\Theta$ can be represented in terms of the $r$ linearly independent columns of $R^{r}$. The coefficients for the columns $\Theta_{k^{\prime}} k=1, \ldots, \alpha_{t^{\prime}}$ in the basis $\left\{R_{j}\right\}_{j=1}^{r}$ are given by the $k^{\text {th }}$ column of $M^{r}$. Since $R$ is orthogonal, we can prove that

$$
\Theta_{k}=\sum_{j=1}^{r}\left\langle\Theta_{k^{\prime}} R_{j}\right\rangle_{\mathbb{R}^{\alpha_{Z}}} R_{j^{\prime}} \text { for } k=1, \ldots, \alpha_{t} .
$$

It follows from SVD that the necessary optimality conditions for Equation (6) are given by the symmetric $\alpha_{Z} \times \alpha_{z}$ eigenvalue problem

$$
\Theta \Theta^{T} \Phi_{p}=C^{\text {right }} \Phi_{p}=\omega_{p} \Phi_{p^{\prime}} \text { for any } p=1, \ldots, r .
$$

The POMs $\left\{\Phi_{p}\right\}_{p=1, \ldots, P}$ can then be determined by computing the eigenvectors (which become matrices after the data-set rearrangement) of the correlation matrix. Note that for applications involving a large number of degrees of freedom, the correlation matrix $\Theta \Theta^{T}$ may become very large and the computation of POMs may rapidly require substantial computational resource.

\section{Penalising benchmark test}

\subsection{Definition of the test}

We considered a penalising case to illustrate a potential POD preprocessing application and check the quality of the heat source estimates. We constructed a heat diffusion problem, which superimposed numerous difficulties, all inspired by physically tricky situations 
accompanying the mechanical tests. More precisely, we chose the following 2D heat diffusion problem on a rectangular domain (i.e. the gauge part of the specimen), with a given heat source distribution $\bar{s}^{h}$ :

$$
\left\{\begin{array}{l}
\rho C\left(\frac{\partial \bar{\vartheta}_{i}(x, y, t)}{\partial t}+\frac{\bar{\vartheta}_{i}(x, y, t)}{\tau_{\mathrm{th}}^{20}}\right)-k_{c}\left(\frac{\partial^{2} \bar{\vartheta}_{i}(x, y, t)}{\partial x^{2}}+\frac{\partial^{2} \bar{\vartheta}_{i}(x, y, t)}{\partial y^{2}}\right)=\bar{s}^{h}(x, y, t) \\
\frac{\partial \bar{\vartheta}_{i}}{\partial x}\left( \pm \frac{L}{2}, y, t\right)=\mp \lambda_{x} \bar{\vartheta}_{i \pm \frac{L}{2}}\left( \pm \frac{L}{2}, y, t\right), \frac{\partial \bar{\vartheta}_{i}}{\partial y}\left(x, \pm \frac{1}{2}, t\right)=\mp \lambda_{y} \bar{\vartheta}_{i \pm \frac{1}{2}}\left(x, \pm \frac{1}{2}, t\right) \\
\bar{\vartheta}_{i}(x, y, 0)=0 .
\end{array}\right.
$$

where the parameters $\lambda_{x}$ and $\lambda_{y}$ were considered as constants.

- The rectangular domain had the dimensions of a standard thin flat sample gauge part of a test specimen. It was $32 \mathrm{~mm}$ long by $25.6 \mathrm{~mm}$ wide, corresponding to a $81 \times 65$ pixel image since the space resolution of the IR camera was about $\Delta x=\Delta y=0.4 \mathrm{~mm}$. The time step $\Delta t$ was $0.1 \mathrm{~s}$ (i.e. frame rate $=10 \mathrm{~Hz}$ ) and 600 thermal fields were computed.

- The thermal boundary conditions were standard Fourier conditions:

$$
-k_{c} \overrightarrow{\operatorname{grad}} \bar{\vartheta} \cdot \vec{n}=h \bar{\vartheta},
$$

where $h$ was a global heat exchange coefficient. We chose a coefficient $h_{x}=k_{c} \lambda_{x}=170$ $\mathrm{W} \mathrm{m}{ }^{2}{ }^{\circ} \mathrm{C}^{-1}$, tenfold greater than $h_{y}=k_{c} \lambda_{y^{\prime}}$ in order to distinguish the heat exchange between the sample and testing machine grips at $x= \pm L / 2$, from the heat exchange with the surrounding air at $y= \pm 1 / 2$.

The value of the time constant associated with heat losses perpendicular to the specimen gauge part was $\tau_{\mathrm{th}}^{2 \mathrm{D}}=200 \mathrm{~s}$. This latter can be assessed using Equation (2) once the material under investigation and the specimen sizes are set.

- The analytical form of the heat source distribution was rather complex. As already mentioned, the goal was to superimpose several heat source patterns, namely:

$\circ$ a homogenous oscillating source $\bar{s}^{\text {the }}$, reflecting the so-called thermoelastic coupling effects (e.g. thermoelastic sources induced by the thermodilitability of materials [42]). This type of source was activated throughout the test duration $\mathcal{D}=60 \mathrm{~s}$. We chose a sinusoidal form $\bar{s}^{\text {the }}=s_{0}^{\text {the }} \sin \left(2 \pi f_{L} t\right)$, where $s_{0}^{\text {the }}$ and $f_{L}$ were constant parameters.

- between $0.1 \mathcal{D}$ and $0.6 \mathcal{D}$, a dissipative source distribution $\bar{s}_{L}^{\text {dis }}$ was programmed, corresponding to the calorimetric manifestation of a Lüders band propagation [13]. This source was written as $\bar{s}_{L}^{\text {dis }}=s_{L}^{0} \exp \left(-a_{L}\left[x-p_{L} y-v_{L}\left(t-t_{L}^{0}\right)\right]^{2}\right)$, where $\left(s_{L}^{0}, a_{L}, p_{L}, v_{L}, t_{L}^{0}\right)$ are constants.

$\circ$ for $t \geq 0.6 D$, a progressive concentration of dissipative source distribution $\bar{s}_{N}^{\text {dis }}$ was introduced. This scenario can be associated with the gradual localisation of plastic strain during necking [11] or with the narrowing of a high dissipation zone induced by fatigue damage heralding the inception of fatigue crack [1]. The form of $\bar{s}_{N}^{\text {dis }}$ was chosen as $\bar{s}_{N}^{\text {dis }}=s_{N}^{0} \widetilde{t}^{2} \exp \left(-a_{N} r^{2}(x, y) /\left[1-\alpha_{N} \tilde{t}\right]\right)$, where $\tilde{t}$ is a dimensionless time ranging from 0 to 1 within $0.6 \mathcal{D}$ and $\mathcal{D}$, and where $r^{2}(x, y)=4\left(x-x_{0}\right)^{2}+\left(y-y_{0}\right)^{2}$, $\left(x_{0}, y_{0}\right)$ are coordinates of the point where the dissipative source gradually concentrates. In Figure 1, a set of six heat source fields is shown. They were computed using the parameter values in Table 1 . Note that sources were divided by $\rho C$ and then expressed in $\left({ }^{\circ} \mathrm{C} \mathrm{s}^{-1}\right)$. 


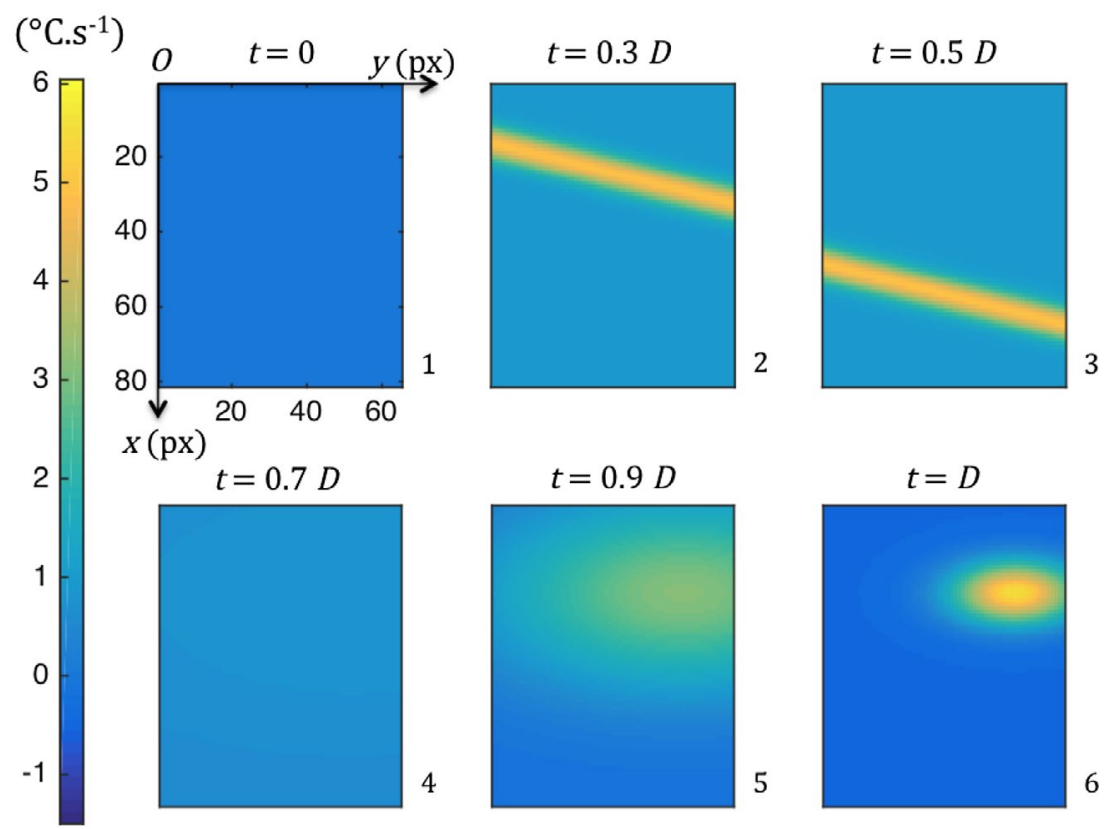

Figure 1. Examples of 2D fields of given heat sources $\bar{s}^{h}$ : images 2,3 show the propagation in the length direction $(O x)$ of an inclined narrow band.

Notes: Images 4-6 show a gradual concentration of heat sources. From 1 to 6 a homogeneous spatial and temporal sinusoidal source is superimposed.

Table 1. Parameter values for the heat source field computations.

\begin{tabular}{|c|c|c|c|c|c|}
\hline $\bar{s}^{\text {the }}$ & $s_{0}^{\text {the }}\left({ }^{\circ} \mathrm{C} \mathrm{s}^{-1}\right)$ & $f_{L}(\mathrm{~Hz})$ & & & \\
\hline & 1 & 0.05 & & & \\
\hline $\bar{s}_{L}^{\text {dis }}$ & $\begin{array}{c}s_{L}^{0}\left({ }^{\circ} \mathrm{C} \mathrm{s}^{-1}\right) \\
4\end{array}$ & $\begin{array}{c}a_{L}\left(\mathrm{~mm}^{2}\right) \\
3.12510^{-3}\end{array}$ & $\begin{array}{c}p_{L} \\
0.25\end{array}$ & $\begin{array}{c}v_{L}\left(\mathrm{~mm} \mathrm{~s}^{-1}\right) \\
1.06\end{array}$ & $\begin{array}{l}t_{L}^{0}(s) \\
120\end{array}$ \\
\hline$\overline{\bar{s}_{L}^{d i s}}$ & $\begin{array}{c}s_{N}^{0}\left({ }^{\circ} \mathrm{C} \mathrm{s}^{-1}\right) \\
6\end{array}$ & $\begin{array}{l}a_{N}\left(\mathrm{~mm}^{-2}\right) \\
6.2510^{-6}\end{array}$ & $\begin{array}{c}a_{N} \\
1.10^{-4}\end{array}$ & $\begin{array}{c}x_{0}(\mathrm{~mm}) \\
9.6\end{array}$ & $\begin{array}{c}y_{0}(\mathrm{~mm}) \\
20.48\end{array}$ \\
\hline
\end{tabular}

- The thermophysical constants of the tested material were also chosen in order to drastically amplify the heat diffusion losses (e.g. $\rho=8290 \mathrm{~kg} \mathrm{~m}^{-3}, \mathrm{C}=385 \mathrm{~J} \mathrm{~kg}^{-1}{ }^{\circ} \mathrm{C}^{-1}$ and $\left.k_{c}=360 \mathrm{~W} \mathrm{~m}^{-1}{ }^{\circ} \mathrm{C}^{-1}\right)$. We chose the properties of pure copper, which has high diffusivity $\left(D=k_{c} / \rho C \approx 10^{-4} \mathrm{~m}^{2} \mathrm{~s}^{-1}\right)$, i.e. dozens of times greater than that of many conventional materials. Note that high diffusivity regularises the temperature fields (in direct problems) and, conversely, scatters the heat source estimates (in inverse problems) because it amplifies numerical errors induced by computation of the Laplacian operator. An order of magnitude of the error associated with the curvature estimates can be given by $\delta \theta /(\delta x)^{2}$, where $\delta \theta$ is the error associated with the thermal data used in the curvature estimates and $\delta x$ is, for instance, the pixel size. To illustrate this idea in the case of copper, a $0.4 \mathrm{~mm}$ space resolution and $0.02{ }^{\circ} \mathrm{C}$ temperature error leads to a heat source error of about $14{ }^{\circ} \mathrm{C} \mathrm{s}^{-1}$. With the same settings, this error would drop to $0.026^{\circ} \mathrm{C} \mathrm{s}^{-1}$ for the polyamide composite mentioned at the end of this paper. 


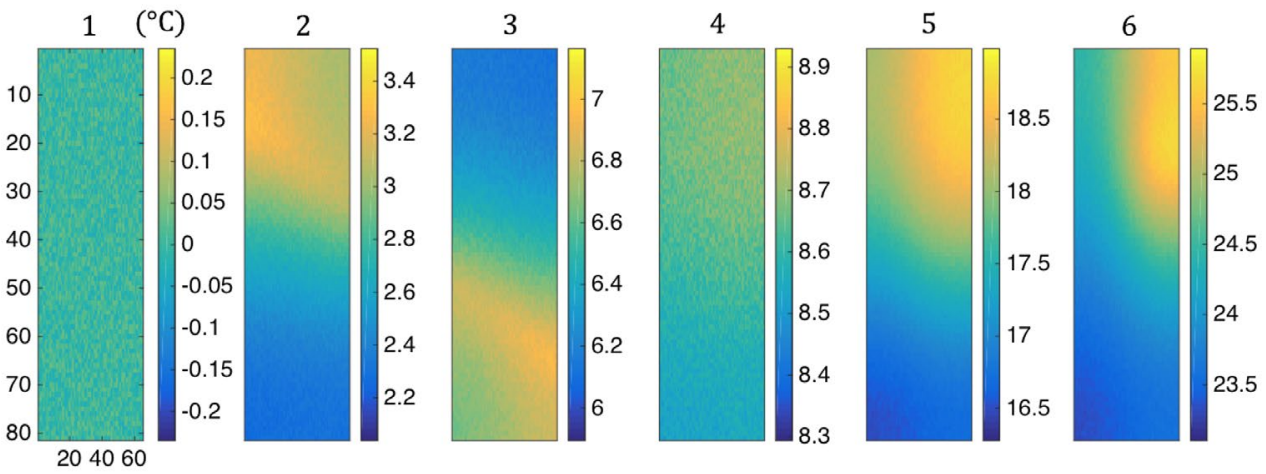

Figure 2. Examples of $\bar{\vartheta}_{r}$ fields.

\subsection{From heat sources to noisy temperature fields}

The solution $\bar{\vartheta}_{i}$ of the heat diffusion problem was computed using a home-made code (MATLAB ${ }^{\circ}$ ) with a simple Euler time integration scheme and finite difference discretization. In accordance with the thermosignal characteristics given by an IRFPA camera, Gaussian white noise $N_{g}$ was superimposed over the temperature variations $\bar{\vartheta}_{i}$ to get a noisy set of thermal images $\bar{\vartheta}_{r}=\bar{\vartheta}_{i}+N_{g}$ (see Figure 2).

In IRT, the extent of noise is characterised by the 'noise equivalence temperature difference' (NETD). For example, NETD $=0.02{ }^{\circ} \mathrm{C}$ means that the camera can detect temperature differences as small as $0.02{ }^{\circ} \mathrm{C}$. NETD expresses the thermal sensitivity of the camera, and good sensitivity allows the user to distinguish objects in a scene that have very little temperature difference between them. Note that current IRFPA cameras provide standard NETDs of about $0.02-0.05^{\circ} \mathrm{C}$. In Figure 2 , we chose a NETD of about $0.07^{\circ} \mathrm{C}$. The six thermal fields correspond to the heat source distributions shown in Figure 1. The high diffusion partly hides the traces of heat source band propagation (fields 2 and 3) and then the progressive localisation (fields 4-7). The instantaneous noise effects can, however, be observed. Note that if a single temperature colour scale had been used for the complete test, neither thermal noise nor temperature gradients at any time would have been visible. That is why a colorbar has been systematically added to each thermal field so as to be able to focus on the temperature range associated with each instant.

During the numerical tests, the following NETD were used: $0.02,0.07,0.12,0.16$ and $0.2{ }^{\circ} \mathrm{C}$. The noisy temperature fields $\bar{\vartheta}_{r}$ were thus used to check the POD preprocessing efficiency.

\subsection{POD preprocessing}

Since the heat diffusion problem is linear with respect to temperature, it is thus possible to use a superposition principle. We then considered the two following sub-problems: the first sub-problem overlooks the heat source distribution $\bar{s}^{h}$ and considers the Dirichlet thermal boundary conditions associated with the initial problem,

$$
\left\{\begin{array}{l}
\rho C\left(\frac{\partial \bar{\vartheta}_{\ell}(x, y, t)}{\partial t}+\frac{\bar{\vartheta}_{\ell}(x, y, t)}{\tau_{\text {th }}^{2 D}}\right)-k_{c}\left(\frac{\partial^{2} \bar{\vartheta}_{\ell}(x, y, t)}{\partial x^{2}}+\frac{\partial^{2} \bar{\vartheta}_{\ell}(x, y, t)}{\partial y^{2}}\right)=0 \\
\bar{\vartheta}_{\ell}\left( \pm \frac{L}{2}, y, t\right)=\bar{\vartheta}_{r}\left( \pm \frac{L}{2}, y, t\right), \bar{\vartheta}_{\ell}\left(x, \pm \frac{1}{2}, t\right)=\bar{\vartheta}_{r}\left(x, \pm \frac{1}{2}, t\right), \\
\bar{\vartheta}_{\ell}(x, y, 0)=0,
\end{array}\right.
$$


while the second sub-problem considers $\bar{s}^{h}$, with homogeneous thermal boundary conditions:

$$
\left\{\begin{array}{l}
\rho C\left(\frac{\partial \bar{\vartheta}_{s}(x, y, t)}{\partial t}+\frac{\bar{\vartheta}_{s}(x, y, t)}{\tau_{\mathrm{th}}^{2 D}}\right)-k_{c}\left(\frac{\partial^{2} \bar{\vartheta}_{s}(x, y, t)}{\partial x^{2}}+\frac{\partial^{2} \bar{\vartheta}_{s}(x, y, t)}{\partial y^{2}}\right)=\bar{s}^{h}(x, y, t), \\
\bar{\vartheta}_{s}\left( \pm \frac{L}{2}, y, t\right)=0, \bar{\vartheta}_{s}\left(x, \pm \frac{1}{2}, t\right)=0, \\
\bar{\vartheta}_{s}(x, y, 0)=0,
\end{array}\right.
$$

where naturally $\bar{\vartheta}_{s}+\bar{\vartheta}_{\ell}=\bar{\vartheta}_{i}+N_{g}=\bar{\vartheta}_{r}$.

Note that the $\bar{\vartheta}_{s}$ field is always achievable once Equation (17) has been numerically solved and the solution $\bar{\vartheta}_{\ell}$ is computed. In practice, the boundary conditions $\bar{\vartheta}_{r}( \pm L / 2, y, t)$ and $\bar{\vartheta}_{r}(x, \pm I / 2, t)$ are given by the IR camera. This so-called lifting operation allows assessment of the heat source distribution via a heat diffusion problem (i.e. Equation (18)) which now has homogeneous boundary conditions. This lifting reduces the complexity of thermal fields coming from possibly complex heat exchanges with the surroundings.

So the POD preprocessing was consequently split into four stages:

(i) The lifting temperature field $\bar{\vartheta}_{e}$ is computed by solving Equation (17) using a simple Euler time integration scheme.

(ii) Once $\bar{\vartheta}_{\ell}$ is computed, $\bar{\vartheta}_{s}$ can be determined via the difference $\bar{\vartheta}_{s}=\bar{\vartheta}_{r}-\bar{\vartheta}_{\ell}$.

(iii) The correlation matrix is then constructed using $\bar{\vartheta}_{s}$ and used to derive the eigenvalues and eigenvectors of the correlation matrix.

(iv) Setting a given number $N_{\text {POD }}$ of eigenvectors (POMs) finally leads to a (filtered) approximation of $\bar{\vartheta}_{s^{\prime}}$ named $\bar{\vartheta}_{\mathrm{POD}}$.

A discrete version of Equation (3) can then be written as $\left(\bar{\vartheta}_{\mathrm{POD}}\right)_{i j}^{k}=\sum_{p=1}^{N_{\text {POD }}} a_{k}^{p} p_{i j}^{p}$ where $\left(\bar{\vartheta}_{\mathrm{POD}}\right)_{i, j}^{k}=\bar{\vartheta}_{\mathrm{POD}}\left(x_{i}, y_{j}, t_{k}\right), a_{k}^{p}=a_{p}\left(t_{k}\right)$ and $P_{i j}^{p}=\Phi_{p}\left(x_{i}, y_{j}\right)$. The scalars $a_{k}^{p}$ represents the projections of the temperature onto the $p$-th POM matrix of components $P_{i j}^{p}$.

In the benchmark test, the size of the correlation matrix was $(65 \times 81)^{2}$, the total number of POMs was then equal to 5265 . Figure 3 just shows the POMs 1, 2, $3 \ldots$ and 14 . If the surface of the first POMs seemed to be smooth and regular, they rapidly became complex (sharp increase in the number of changes of curvature sign) and looked like a noise effect induced by the finite space discretization.

Recall that with a great number of POMs the signal and its noise is, by construction, reconstructed (Equation (3)). The challenge of using a small set of POMs is naturally to reconstruct (to filter) the thermosignal limiting the noise effects. Here, 14 POMs were necessary, as shown in the next subsection.

The POD prefiltering efficiency can thus be illustrated by computing the temperature differences between $\bar{\vartheta}_{i}-\bar{\vartheta}_{\ell}=\bar{\vartheta}_{s}-N_{g}$ on the one hand, and $\bar{\vartheta}_{\text {POD }}$ on the other (see Figure 4).

These differences simultaneously show the noise reduction induced by POD filtering on the lifted temperature fields (random error), but also the relevance of the projection of the temperature fields onto the orthonormal POD basis (approximation error). Figure 4 indeed shows that the maximum residues were less than $0.02{ }^{\circ} \mathrm{C}$. The mean value and the standard deviation associated with the 600 fields of the whole numerical test were about $6.810^{-4}$ and $6.810^{-3}{ }^{\circ} \mathrm{C}$, respectively. The quasi-centred distribution of temperature differences showed an absence of systematic error, while the order of magnitude of the standard deviation 
POM \#1

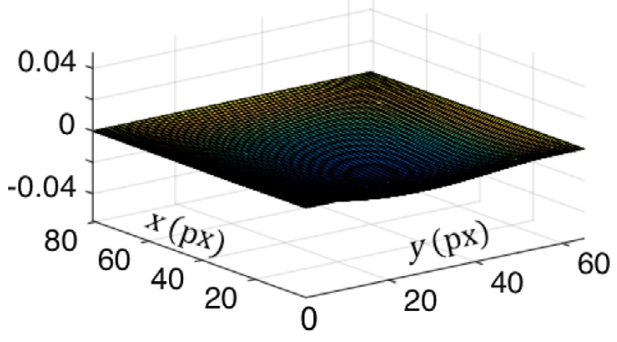

POM \#3

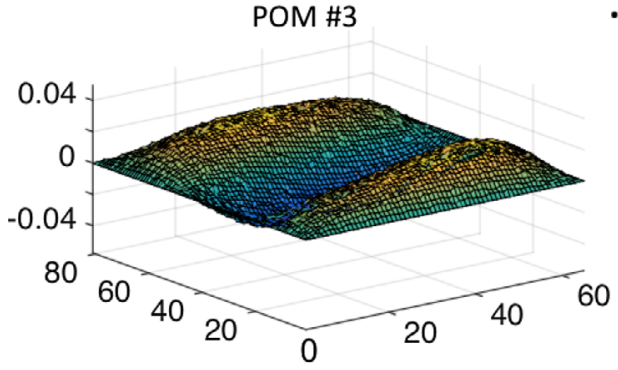

POM \#2

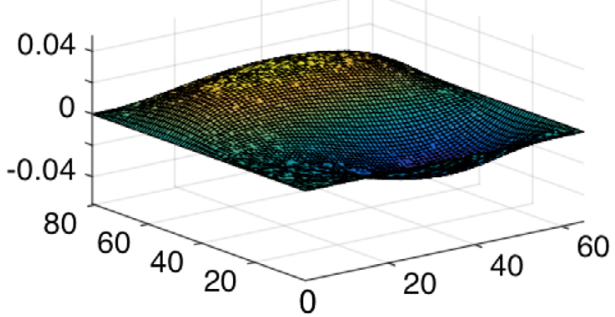

POM \#14

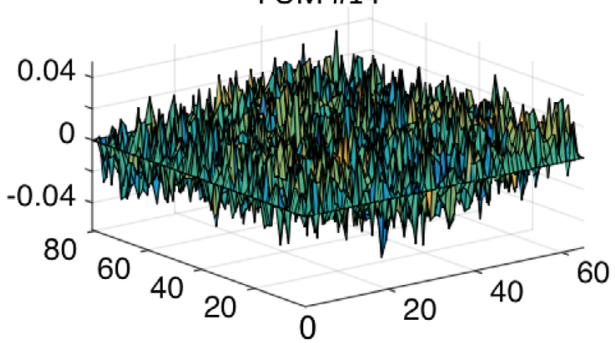

Figure 3. First normalised proper orthogonal modes (POMs) associated with the benchmark test.

$\left({ }^{\circ} \mathrm{C}\right)$

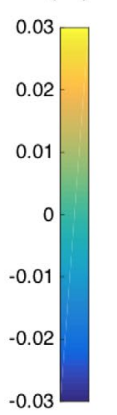

1

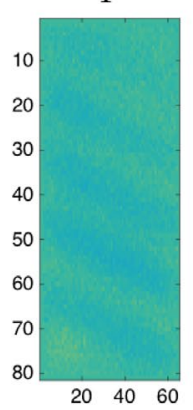

2

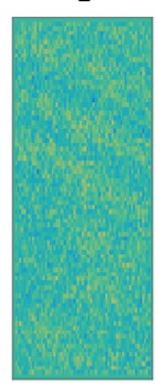

4

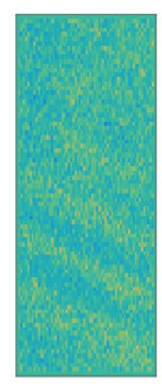

5

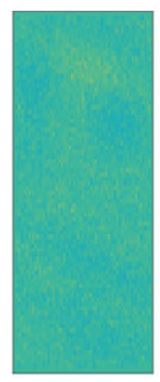

6

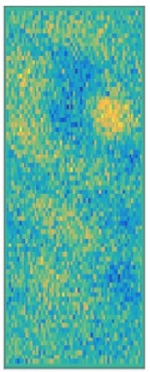

Figure 4. Examples of 2D fields of the temperature difference $\bar{\vartheta}_{i}-\bar{\vartheta}_{\ell}-\bar{\vartheta}_{\text {POD}}$.

indicated a substantial noise reduction induced by the sole use of 14 POMs to reconstruct the temperature fields.

To complete the POD preprocessing illustration, Figure 5 gives the time course of the components $a_{p}(t), p=1, . ., 5$.

A gradual decrease in the $a_{p}$ amplitude can first be observed. As the temperature fields increase in spatial complexity and temporally change, this amplitude continues to decrease but at a slower rate.

The benchmark test was constructed to get this slow decrease introducing heterogeneous, moving in space and varying in time heat source distributions, for a very highly diffusive material. At least 14 POMs were required to properly reconstruct the details of the 


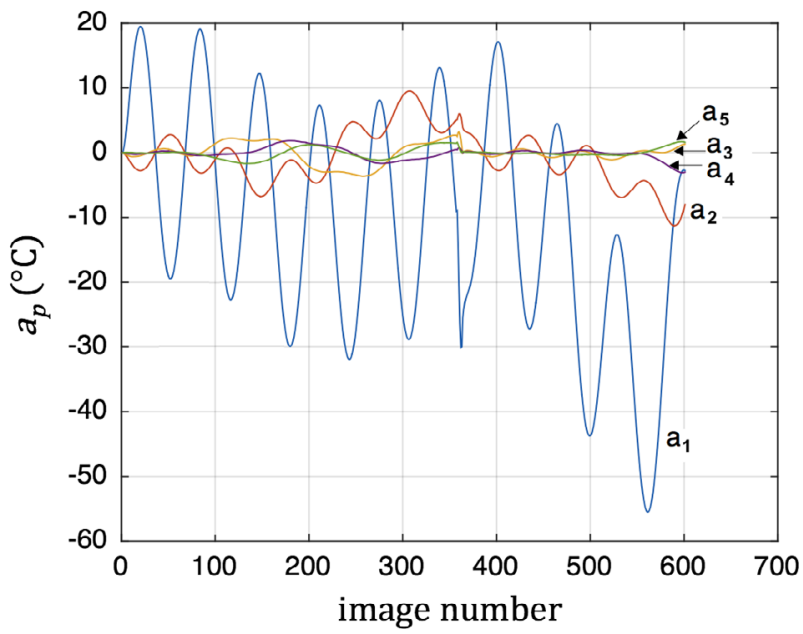

Figure 5. Time course of the first five components $a_{p}(t)$ of $\bar{\vartheta}_{\mathrm{POD}}$.
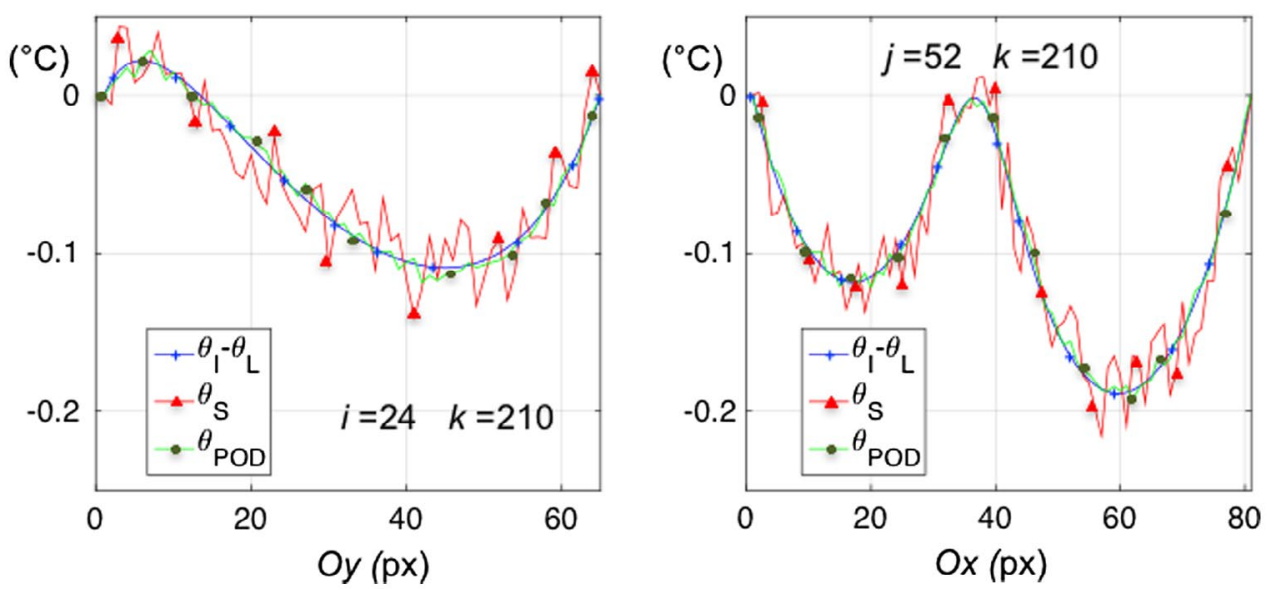

Figure 6. Examples of temperature profiles: comparison of $\bar{\vartheta}_{i}-\bar{\vartheta}_{\ell}$ (pure lifted solution-(blue + )), $\bar{\vartheta}_{s}$ (noisy lifted solution-(red $\mathbf{\Delta})$ ) and $\bar{\vartheta}_{\text {POD }}$ (approximated solution-(green $\left.\bullet\right)$ ).

temperature fields derived from these heat sources fields. However, the extent of noise reintroduced in the signal increases with the number of POMs.

Finally, it is worth noting that the influence of the oscillating source $\bar{s}^{\text {the }}$ throughout the temperature variation test is reflected by the time course of $a_{1}$ and probably $a_{2}$, with the oscillation period being roughly the same.

Finally, to check the reliability of temperature approximation by the POD technique, Figure 6 gives the longitudinal and transversal profiles passing through $M_{0}\left(x_{0}, y_{0}\right)$, i.e. pixel ( $i=24, j=52$ ). We chose a particular instant when the signal-to-noise ratio was particularly $\operatorname{bad}\left(t_{k}=0.35 D\right.$, i.e. $\left.k=210\right)$. The three profiles correspond to $\bar{\vartheta}_{i}-\bar{\vartheta}_{\ell}, \bar{\vartheta}_{s^{\prime}}$ and $\bar{\vartheta}_{\mathrm{POD}}$. The qualities of the POD approximation underlined in Figure 4 can once again be verified. 
Although, for this pathological case, the use of 14 POMs is satisfactory to reconstruct the pure lifted solution, a small part of the noise is nevertheless preserved. Recall that one main goal of the image processing is to estimate the second derivatives (curvatures) of the temperature to assess the heat source fields. Here we can see the limitations of POD use. If the number of POMs is reduced, the noise influence decreases but the approximation errors may consequently increase. At this level, it would be interesting to discuss the efficiency of the POD preprocessing technique by varying the noise amplitude and number of POMs.

\subsection{Efficiency of POD preprocessing}

For this purpose, we introduced the dimensionless $Z$ ratio, defined as:

$$
Z=\sqrt{\widehat{\Theta^{2}}} / \widehat{\delta \Theta^{2}}
$$

where $\widehat{\delta \Theta^{2}}$ stands for the spatiotemporal average of the differences $\left(\bar{\vartheta}_{\mathrm{POD}}-\left(\bar{\vartheta}_{i}-\bar{\vartheta}_{\ell}\right)\right)^{2}$, while $\widehat{\Theta^{2}}$ represents the spatiotemporal average of the squared lifted temperature $\left(\bar{\vartheta}_{i}-\bar{\vartheta}_{\ell}\right)^{2}$. This statistical 'signal-to-error' ratio expresses the capability of the POD techniques to approximate and filter the thermal data. Its value naturally depends on the set of thermal data considered to compute the ratio itself. To give examples of $Z$ values in some pathological cases, we considered the profiles given in Figure 6. The lifted temperatures were particularly low in this case. We found $Z$ values of about $12-13$ for the $\bar{\vartheta}_{\text {POD }}$ profiles and of about 3-4 when considering the $\bar{\vartheta}_{s}$ profiles instead of $\bar{\vartheta}_{\text {POD }}$.

More systematically, Figure 7 depicts variations in the $Z$ ratio as a function of the number of POMs and NETD for the whole test. A rapid overview showed that, at constant NETD, the $Z$ intensity rapidly increased as a function of the number of POMs and reached an asymptotic value. As already mentioned, 14 POMs were sufficient to get these maximum $Z$ values and obtain an excellent approximation of $\bar{\vartheta}_{s}$ (as shown in Figure 6). These asymptotic values started at 44 for zero noise and decreased slightly to 24 for NETD $=0.2^{\circ} \mathrm{C}$. In such cases, the decrease in $Z$ was solely due to the thermal noise. Conversely, for a small number of POMs, Figure 7 still shows that the influence of the NETD on the $Z$ ratios remained negligible. In such cases, the approximation errors were substantially greater than errors induced by the thermal noise.

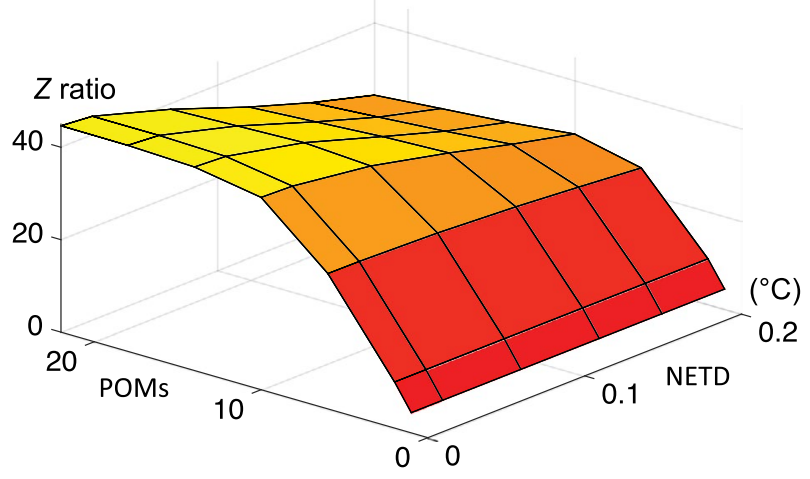

Figure $7 . Z$ ratio map with respect to the number of POMs and NETD values. 


\subsection{From temperature to heat source fields}

The ultimate goal is now to check if POD pre-processing is sufficiently effective to directly derive heat sources from the $\bar{\vartheta}_{\text {POD }}$ fields by simply using a finite difference approximation.

We have already mentioned that the tricky point was to estimate Laplacian terms depicting local heat losses by conduction. The reliability of heat source derivations using POD prefiltering is illustrated in Figure 8. Again considering the particular case $(t=0.35 D$, i.e. $k=210)$ where the signal-to-noise ratio was particularly bad (see Figure 6), Figure 8 shows (left column) the $\bar{\vartheta}_{\text {POD }}$ field and the corresponding $\Delta \bar{\vartheta}_{\text {POD }}$ field computed using finite differences. Clearly the residual noise on $\bar{\vartheta}_{\text {POD }}$ obtained with 14 POMs here generated Laplacians that were too noisy to be directly used in the heat source computation. However, the right column shows that a slight and rapid filtering operation via a Gaussian kernel is sufficient to provide a $\widetilde{\vartheta}_{\text {POD }}$ field whose Laplacians $\Delta \widetilde{\vartheta}_{\mathrm{POD}}$ are now regular.

The computed heat sources $\widetilde{s}_{\text {POD }}$ were presented in Figure 9 . They were computed using a simple finite difference approximation of the heat diffusion equation and $\widetilde{\vartheta}_{\text {POD }}$ fields:

$$
\left(\tilde{s}_{\mathrm{POD}}\right)_{i, j}^{k}=\frac{\left(\tilde{\vartheta}_{\mathrm{POD}}\right)_{i, j}^{k}-\left(\tilde{\vartheta}_{\mathrm{POD}}\right)_{i, j}^{k-1}}{\Delta t}+\frac{\left(\tilde{\vartheta}_{\mathrm{POD}}\right)_{i, j}^{k}}{\tau_{\mathrm{th}}^{2 \mathrm{D}}}-\frac{k_{c}}{\rho C}\left(\Delta \tilde{\vartheta}_{\mathrm{POD}}\right)_{i, j}^{k}
$$

where:

$$
\left(\Delta \tilde{\vartheta}_{\mathrm{POD}}\right)_{i, j}^{k}=\frac{\left(\tilde{\vartheta}_{\mathrm{POD}}\right)_{i+1, j}^{k}-2\left(\tilde{\vartheta}_{\mathrm{POD}}\right)_{i, j}^{k}+\left(\tilde{\vartheta}_{\mathrm{POD}}\right)_{i-1, j}^{k}}{\Delta x^{2}}+\frac{\left(\tilde{\vartheta}_{\mathrm{POD}}\right)_{i, j+1}^{k}-2\left(\tilde{\vartheta}_{\mathrm{POD}}\right)_{i, j}^{k}+\left(\tilde{\vartheta}_{\mathrm{POD}}\right)_{i, j-1}^{k}}{\Delta y^{2}}
$$
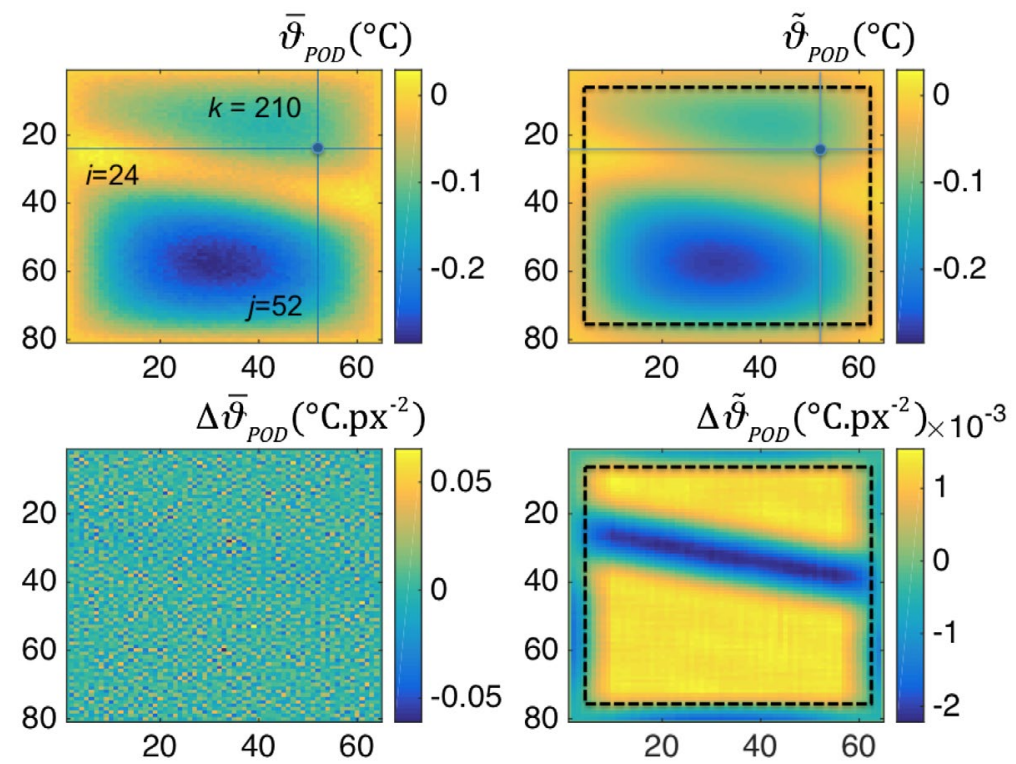

Figure 8. Left column: fields of $\bar{\vartheta}_{\text {POD }}$ and $\Delta \bar{\vartheta}_{\text {POD }}$ at $t=0.35 \mathrm{D}$. Right column: field of $\tilde{\vartheta}_{\text {POD }}$ slightly smoothed via a Gaussian convolutive filter and field of $\Delta \widetilde{\vartheta}_{\mathrm{POD}}$. Outside the dashed rectangle, zero-padding was used to perform the convolution. 


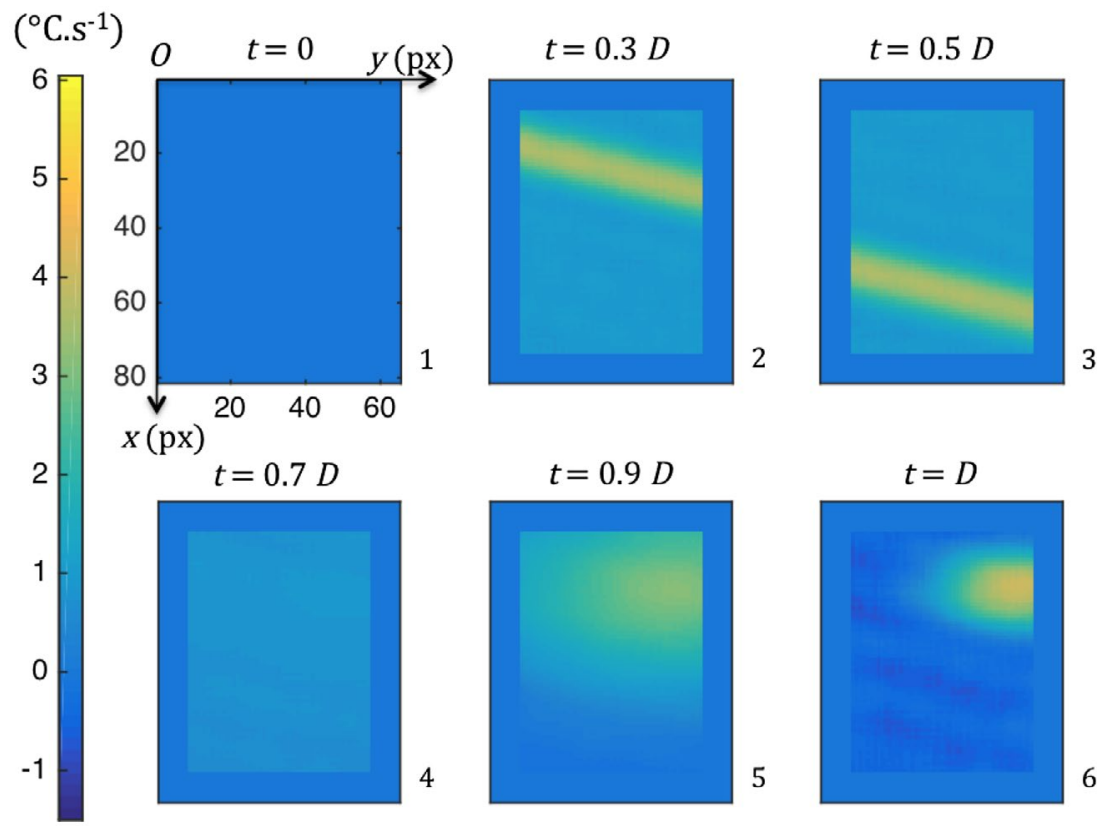

Figure 9. Fields of the computed heat sources $\tilde{s}_{\text {POD }}(P O M s=14, N E T D=0.07)$. Note: Reconstructed source fields should be compared to those in Figure 1.
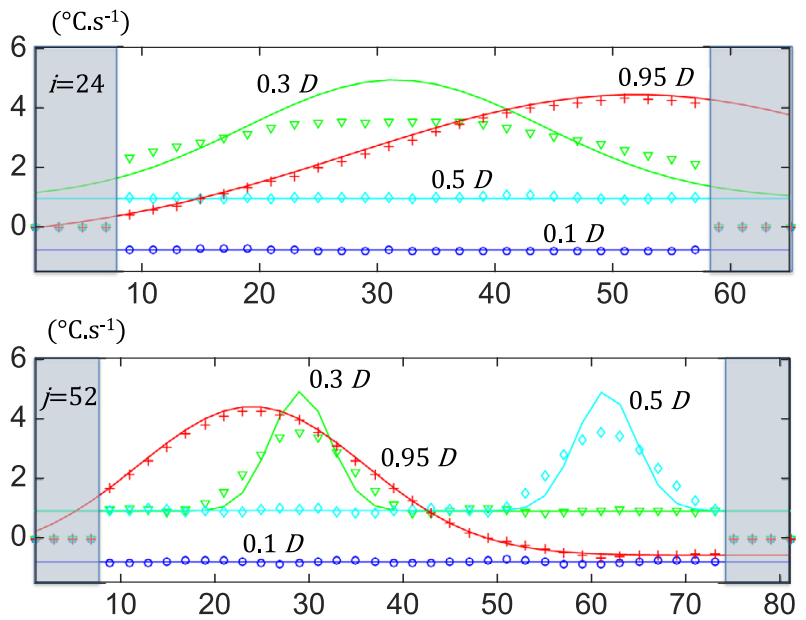

Figure 10. Profiles of heat source fields passing through $\left(x_{0}, y_{0}\right)$ at four different times.

Notes: Full lines represent the given heat sources $\bar{s}^{h}$ while symbols (circles, crosses, etc.) give the reconstructed values $\tilde{s}_{\text {POD }}^{h}$.

In Figure 9, the different stages observed in Figure 1 can be easily detected: in images 2 and 3 the propagation of an inclined narrow band of heat sources can be recognised in the length direction $(O x)$. Images 4-6 show a gradual concentration of heat sources around $\left(x_{0^{\prime}} y_{0}\right)$. The main difference with regard to the original heat source distributions was a crushing of localisation zones, inducing a spread of heat source intensities. This classical crushing due to convolutive filtering is clearly visible in Figure 10, where horizontal and vertical heat source 

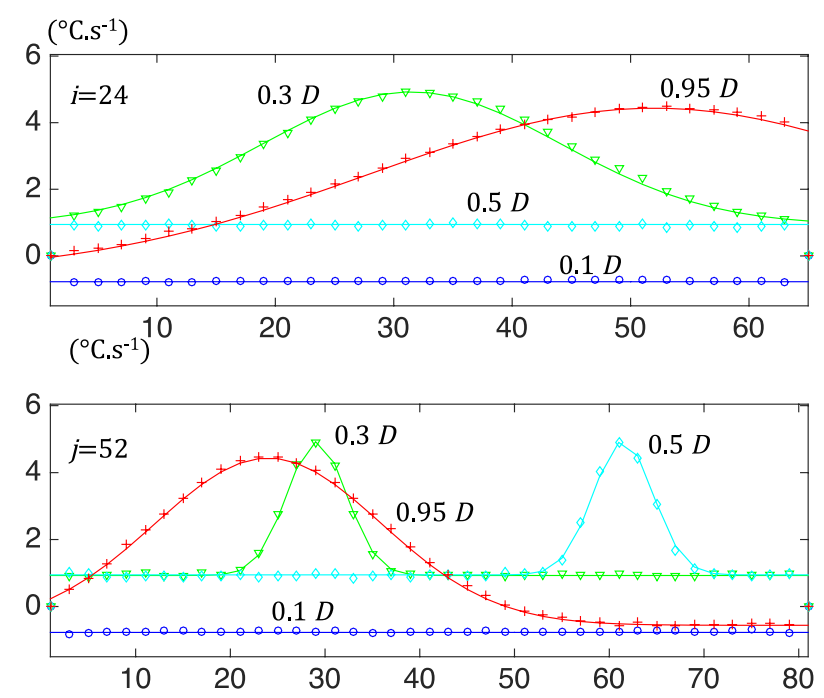

Figure 11. Profiles of heat source fields passing through $\left(x_{0^{\prime}} y_{0}\right)$ at four different times (case of polyamide). Notes: Full lines represent the given heat sources $\bar{s}^{h}$ while symbols (circles, crosses, etc.) give the reconstructed values $\tilde{s}_{\text {POD }}^{h}$ $(\mathrm{POMS}=28, \mathrm{NETD}=0.07)$.

distributions, associated with the profiles defined in Figures 6 and 8, were plotted. Full lines were used to plot the given heat sources $\bar{s}^{h}$, symbols (e.g. circle, cross, ...) depicting $\tilde{s}_{\text {POD }}^{h}$ values. The correspondence between given and reconstructed heat source values were quasi-perfect, except in the vicinity of regions where there was marked curvature in the heat source field (i.e. propagation of the narrow band of heat sources).

At this level, we would like to again stress the pathological character of the benchmark test, which we propose here to show the limitations of the method. It must be clear that in the case of a more standard thermal diffusivity, the errors done on the curvature of the temperature fields would be less important. The cutoff frequency of the filter could then be increased allowing a better catch of the heat source field curvatures without increasing too much the noise on heat source assessments. In many situations (e.g. low diffusivity materials), convolutive filtering is no longer useful and a direct heat source field estimate can be done just using $\tilde{\vartheta}_{\text {POD }}$. As a proof in Figure 11, the benchmark test was performed using the thermophysical properties of the polyamide $\left(\rho=1200 \mathrm{~kg} \mathrm{~m}^{-3}, \quad C=1600 \mathrm{~J} \mathrm{~kg}^{-1}{ }^{\circ} \mathrm{C}^{-1}\right.$, $k_{\mathrm{c}}=0.4 \mathrm{~W} \mathrm{~m}^{-1}{ }^{\circ} \mathrm{C}^{-1}$ ), while not changing the other adjustable test parameters. Only the number of POMs were increased up to POMs $=28$ to avoid high approximation errors. Indeed, low diffusivity induces an increase in the temperature field curvatures and temperature variations for the same heat source distribution and thermal boundary conditions.

The given and reconstructed heat source profiles are quasi-indistinguishable in Figure 11.

\section{POD preprocessing applied at an experimental test}

We checked the efficiency of POD preprocessing applied in standard experimental tests through a cyclic fatigue test on a composite polyamide that is widely used in automotive industries to reduce to the weight of cars [20]. 


\subsection{Experimental context}

PA6.6 was here reinforced with an E-glass fibre content of $30 \%$ by weight. The fibre orientation angle was $45^{\circ}$ with respect to the loading direction. Tensile-tensile cyclic tests were conducted using an MTS hydraulic testing machine equipped with a $\pm 25 \mathrm{kN}$ load cell. The load ratio and the loading frequency were 0.1 and $10 \mathrm{~Hz}$, respectively. Thin flat specimens were used with a gauge part volume of $20 \times 14 \times 3.14 \mathrm{~mm}^{3}$. The mean thermal diffusivity and the time constant $\tau_{\text {th }}^{2 \mathrm{D}}$ introduced in Equation (1) were separately identified. The following mean values were obtained: $D_{c} \approx k_{c} / \rho C \approx 1.81 \times 10^{-7} \mathrm{~m}^{2} \mathrm{~s}^{-1}$ and $\tau_{\mathrm{th}}^{2 \mathrm{D}} \approx 200 \mathrm{~s}$ corresponding to a heat exchange coefficient $h$ of about $13 \mathrm{~W} \mathrm{~m}^{-2}{ }^{\circ} \mathrm{C}^{-1}$ [20]. As already underlined, this situation is considerably easier to deal with than with copper in the previous benchmark test. Here the heat diffusion of the material was low (552-fold lower than that of pure copper), thus reducing the impact of Laplacian assessment errors in the heat source estimates.

The experimental setup involved a focal plane array infrared camera. During the tests, the infrared device was placed in front of the specimen, with the lens axis set perpendicular to the specimen surface. The infrared camera was calibrated using a black body. Readers interested in the complex metrological aspects of IR techniques are referred to $[1,13,39]$.

The spatial resolution of the IR camera was $\Delta x=\Delta y=0.357 \mathrm{~mm}$, with an image size of $77 \times 39$ pixels. A set of 13622 images was recorded at 100 frames per second.

The temperature variations, measured at the specimen surface, were assumed to remain close to the mean depthwise temperature variations over the sample thickness $e$. This assumption was especially relevant since the sources were regularly distributed over $e$ and the Biot number $B_{i} \approx h e / k_{c}$ was small compared to the unit. A Biot number of $10^{-2}$ was calculated in this case, inducing small heat exchanges by convection with the surroundings and consequently small temperature gradients at the sample surface.

\subsection{Thermal image preprocessing}

We first used the POD preprocessing technique to filter the $\tilde{\vartheta}_{r}$ fields. Figure 12 shows image \# 900 of the test. On the left side, the noisy temperature variation field $\tilde{\vartheta}_{r}$ can be observed
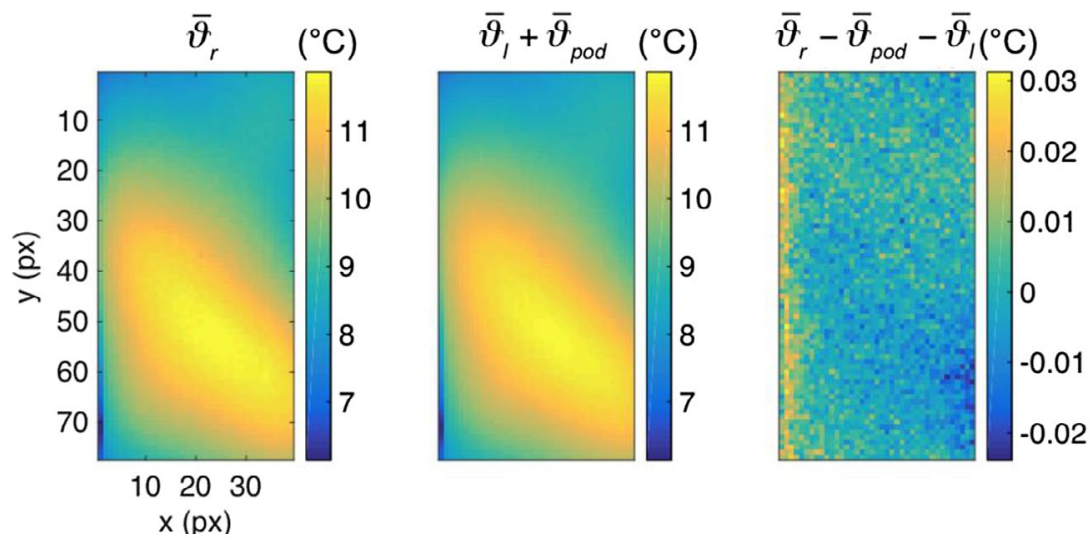

Figure 12. Comparison of $\bar{\vartheta}_{r}$ (left) and $\bar{\vartheta}_{\rho}+\bar{\vartheta}_{\text {POD }}$ (centre) fields.

Notes: The $\delta \bar{\vartheta}$ field (right) shows the random character of the residue obtained with just six POMs. A noise amplitude close to the NETD of the IR camera can be observed. 
and compared to the field $\tilde{\vartheta}_{l}+\tilde{\vartheta}_{\mathrm{POD}}$. On the right, the difference $\delta \tilde{\vartheta}=\tilde{\vartheta}_{r}-\tilde{\vartheta}_{\mathrm{POD}}-\tilde{\vartheta}_{l}$ is also plotted. Note that this difference is the same order of magnitude as the NETD of the camera $\left(\mathrm{NETD}=0.02^{\circ} \mathrm{C}\right)$. Only six POMs were useful for correctly approximating the noisy temperature fields throughout the test and drastically reduced the noise amplitude.

\subsection{Heat source assessments}

In thermographic analysis of material fatigue, the ultimate objective of thermal data processing is to derive an average dissipation field per cycle involved in the gauge part of the specimen under investigation. Indeed, this average operation per cycle allows to eliminate thermomechanical coupling effects (which vanish over a cycle) and then the mean dissipation, representative of the material degradation kinetic throughout the (often long) fatigue test, can be obtained. Considering that the microstructure degradation during a small number $n$ of cycles is low, the intrinsic dissipation source $\tilde{d}_{1}$ can in practice be computed using the following definition:

$$
\tilde{d}_{1}=\tilde{s}^{h}=\oint_{\text {ncycles }} n^{-1} f_{L} \bar{s}^{h} d \tau
$$

where $f_{L}$ stands for the loading frequency and $n \approx 200$.

The mean intrinsic dissipation fields per cycle were computed using both a standard local polynomial fitting based on least-squares techniques (denoted $\tilde{s}_{I s q}^{h}$ ), and the POD preprocessing method (denoted $\tilde{s}_{\text {POD }}^{h}$ in Figure 13). Although a detailed analysis of these results is not within the scope of this paper, we noticed that the spatial distributions of these dissipative fields were not uniform and varied similarly regardless of the data processing approach used.

The residue $\delta \tilde{s}^{h}=\tilde{s}_{\text {Isq }}^{h}-\tilde{s}_{\mathrm{POD}}^{h}$ remained very small for all of the selected images (less than $0.01^{\circ} \mathrm{C} \mathrm{s}^{-1}$ ) while slightly increasing throughout the cyclic loading because of the progressive concentration of heat sources.

\subsection{Image processing times}

Last but not least, the overall computation times of both approaches to process the thermal data file (here about $975 \mathrm{Mo}$ ) were computed. Indeed, it was interesting to compare the time necessary to filter the temperature fields and assess the corresponding heat source distributions. The use of POD processing considerably reduced ( 13 $\mathrm{min})$ the processing time compared times required for a standard local least squares fitting ( $\sim 3.5$ months). The time savings represented about $10^{4}$, which is of paramount importance for research and development services which generally have many fatigue tests to process to identify the lifespan criteria. Note finally that in both cases, the memory requirements on a PC with an Intel Core i7 $2.5 \mathrm{GHz}$ processor with $16 \mathrm{~GB}$ of RAM were about $5 \mathrm{~GB}$ for the given example.

\section{Conclusions}

In this study, POD techniques were applied to filter 2D thermal fields before assessing the associated heat source distribution. The main goal was to achieve rapid derivation of the 

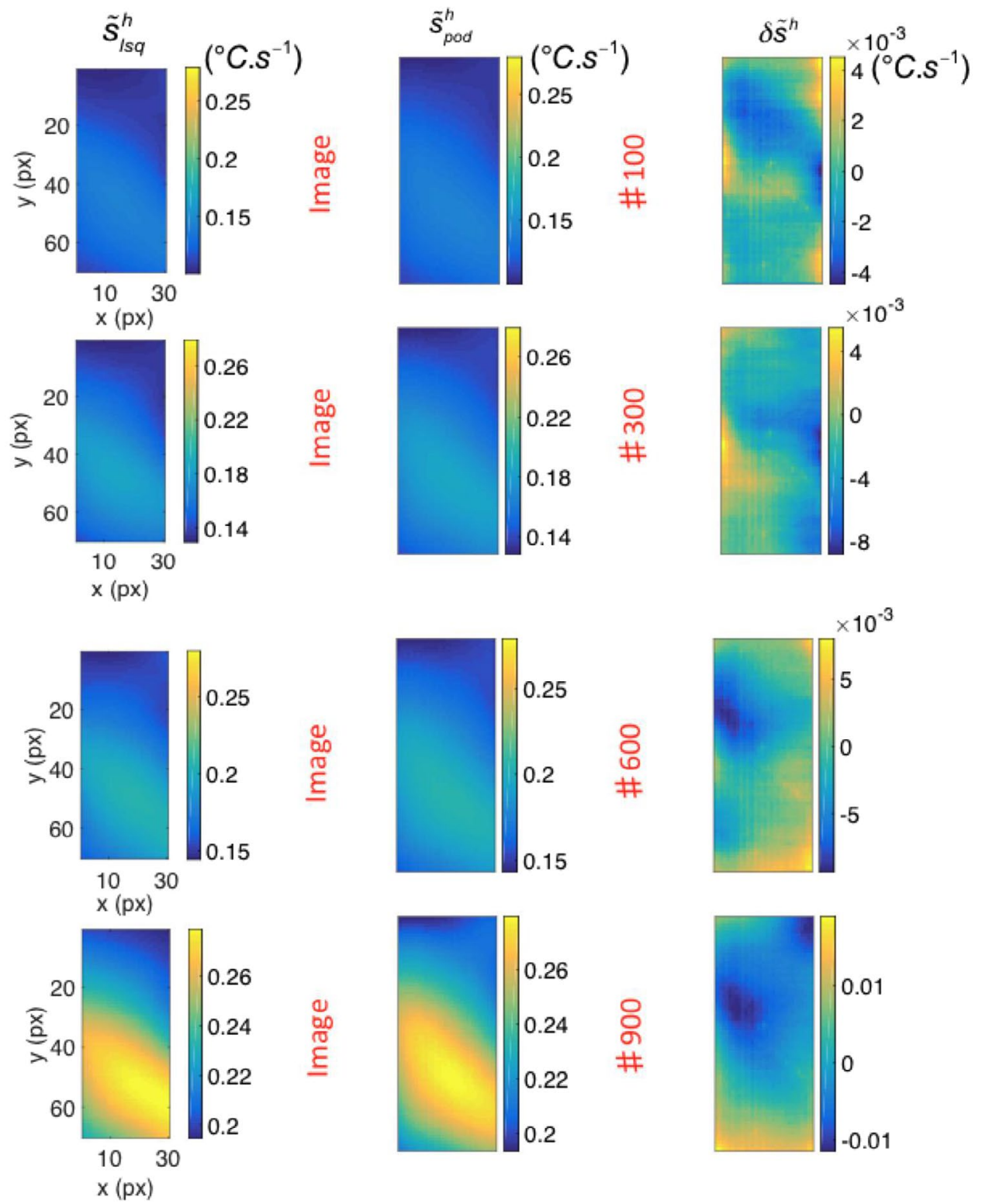

Figure 13. Heat source fields $\tilde{s}_{I s q}^{h}$ and $\tilde{s}_{P O D}^{h}$ of mean intrinsic dissipation per cycle captured at four different time steps corresponding to images \# 100, 300, 600, 900.

heat sources from noisy thermal data. A penalising benchmark test was considered for theoretical validation of the POD method. A high diffusion material was thus chosen in order to regularise the temperature fields induced by heat sources and then, conversely, to complexify the inverse heat source estimates from thermal data. A systematic analysis of approximation errors and thermal noise induced errors was performed. For a given distribution of heat sources and boundary conditions, a decrease in approximation errors was observed. A minimal number of POMs could be identified for each source distribution to achieve an 
optimal reconstruction of thermal fields. In parallel, an increase in noise errors was noted with an increase in the number of POMs, with the noise being gradually taken into account in the POM approximation. Different penalising NETD were also taken into account to check the POD prefiltering robustness. We confirmed that the disturbing effects of standard thermal noise on the heat source computation could satisfactorily be controlled once an optimal number of POMs (limiting the approximation errors) was defined. For the pathological case of copper, convolutive filtering was necessary to compute reliable heat source fields. However, a simple finite difference computation of the heat source fields using the approximated temperature fields by POD are generally sufficient to get satisfactory results since low heat diffusion materials are considered.

As an illustrative example, an application of POD prefiltering to several temperature fields extracted from a fatigue test on a composite polyamide was presented. Its heat diffusion coefficient was particularly low. Local fields of mean intrinsic dissipation per cycle were computed at different loading stages using a simple finite difference approximation and local least-squares fitting. We found that both distributions matched very well throughout the test. We also highlighted that POD led to tremendous savings in computation time compared with local least-squares fitting methods. Indeed, the POD method reduced the processing time by around $10^{4}$-fold!

\section{Acknowledgements}

The authors gratefully acknowledge Solvay Engineering Plastics for providing specimens and the Durafip group, led by Dr Gilles Robert, for fruitful discussions over the last 4 years. The authors would also like to warmly thank the anonymous reviewers for their very constructive comments and complementary suggestions, which led us to deeply reconsider this paper in both its form and content.

\section{Disclosure statement}

No potential conflict of interest was reported by the authors.

\section{References}

[1] Boulanger T, Chrysochoos A, Mabru C, et al. Calorimetric analysis of dissipative and thermoelastic effects associated with the fatigue behavior of steels. Int J Fatigue. 2004;26:221-229.

[2] Chrysochoos A, Huon V, Jourdan F, et al. Use of full-field digital image correlation and infrared thermography measurements for the thermomechanical analysis of material behavior. Strain. 2010;46:117-130.

[3] Chrysochoos A, Peyroux R. Experimental analysis and numerical modeling of thermomechanical couplings in solid materials (in French). Rev Gén Thermique. 1998;37:582-606.

[4] Chrysochoos A, Louche $\mathrm{H}$. An infrared image processing to analyse the calorific effects accompanying strain localization. Int J Eng Sci. 2000;38:1759-1788.

[5] Balandraud X, Chrysochoos A, Leclercq S, et al. Influence of the thermomechanical coupling on the propagation of a phase change front. CR Acad Sci Ser Ilb: Mec. 2001;329:621-626.

[6] Pottier T, Louche H, Samper S, et al. Proposition of a modal filtering method to enhance heat source computation within heterogeneous thermomechanical problems. Int J Eng Sci. 2014;81:163-176.

[7] Delpueyo D, Balandraud X, Grédiac M. Heat source reconstruction from noisy temperature fields using an optimized derivative Gaussian filter. Infrared Phys Technol. 2013;60:312-322.

[8] Boulanger T. Infrared thermography analysis of heat sources induced by steel fatigue [thesis in French]. Montpellier: University of Montpellier II, Sciences et Techniques du Languedoc; 2014. 
[9] Wattrisse B, Muracciole J-M, Chrysochoos A. Thermomechanical effects accompanying the localized necking of semi-crystalline polymers. Int J Therm Sci. April 2002;41:422-427.

[10] Muracciole J-M, Wattrisse B, Chrysochoos A. Thermomechanical analysis of the yield behavior of semi-crystalline polymer. In: Proceedings of the 13th International Conference on Experimental Mechanics: Experimental Analysis of Nano and Engineering Materials and Structures, Editor Gdoutos E.-E; 2007, Alexandroupolis: Springer Netherlands; p. 871-872. doi:10.1007/978-1-40206239-1_433

[11] Chrysochoos A, Wattrisse B, Muracciole J-M, et al. Fields of stored energy associated with localized necking of steel. J Mech Mater Struct. 2009;4:245-262.

[12] Renault N, André S, Maillet D, et al. A spectral method for the estimation of a thermomechanical heat source from infrared temperature measurements. Int J Therm Sci. 2010;49:1394-1406.

[13] Louche H, Chrysochoos A. Thermal and dissipative effects accompanying Lüders band propagation. Mater Sci Eng A. 2001;307:15-22.

[14] Benaarbia A, Chrysochoos A, Robert G. Fiber orientation effects on heat source distribution in reinforced polyamide 6.6 subjected to low cycle fatigue. J Eng Math. 2014;90:13-36.

[15] Louche H, Schlosser P, Favier D, et al. Heat source processing for localized deformation with nonconstant thermal conductivity: application to superelastic tensile tests of NiTi shape memory alloys. Exp Mech. 2012;52:1313-1328.

[16] Favier D, Louche $\mathrm{H}$, Schlosser P, et al. Homogeneous and heterogeneous deformation mechanisms in an austenitic polycrystalline Ti-50.8 at \% Ni thin tube under tension. Investigation via temperature and strain fields measurements. Acta Mater. 2007;55:5310-5322.

[17] Peyroux R, Chrysochoos A, Licht C, et al. Thermomechanical couplings and pseudoelasticity of shape memory alloys. Int J Eng Sci. 1998;36:489-509.

[18] Delpueyo D, Grédiac M, Balandraud X, et al. Investigation of martensitic microstructures in a monocrystalline $\mathrm{Cu}-\mathrm{Al}-\mathrm{Be}$ shape memory alloy with the grid method and infrared thermography. Mech Mater. 2012;45:34-51.

[19] Schlosser $P$, Louche $H$, Favier $D$, et al. Image processing to estimate the heat sources related to phase transformations during tensile tests of NiTi tubes. Strain. 2007;43:260-271.

[20] Benaarbia A, Chrysochoos A, Robert G. Thermomechanical behavior of PA6.6 composites subjected to low cycle fatigue. Composites Part B: Eng. 2015;76:52-64.

[21] Muracciole J-M, Wattrisse B, Chrysochoos A. Energy balance of a semi-crystalline polymer during local plastic deformation. Strain. 2008;44:468-474.

[22] Ranc N, Blanche A, Ryckelynk D, et al. POD preprocessing of IR thermal data to assess heat source distributions. Exp Mech. 2014;55:725-739.

[23] Phung N-L, Marti N, Blanche A, et al. Very high cycle fatigue for single phase ductile materials: slip band appearance criterion. 5th Fatigue Design Conference, Fatigue Design. Senlis. Elsevier. 2013;66:616-625.

[24] Rosenfeld A, Kak A. Digital picture processing. New York (NY): Academic Press; 1982.

[25] Algazi V, Sakrison D. On the optimality of Karhunen-Loève expansion. IEEE Trans. Inf. Theory. 1969;15:319-321.

[26] Holmes P, Lumley J, Berkooz G. Turbulence, coherent structures, dynamical systems and symmetry. Cambridge: Cambridge University Press; 1996.

[27] Berkooz G, Holmes P, Lumley J-L. The proper orthogonal decomposition in the analysis of turbulent flows. Annu Rev Fluid Mech. 1993;25:539-575. doi:10.1146/annurev.fl.25.010193.002543

[28] Graham M, Kevrekidis I. Alternative approaches to the Karhunen-Loève decomposition for model reduction and data analysis. Comput Chem Eng. 1996;20:495-506.

[29] Shvartsman S, Kevrekidis I. Low-dimensional approximation and control of periodic solutions in spatially extended systems. Phys Rev E. 1998;58:361-368.

[30] Shvartsman S, Theodoropoulos C, Rico-Martinez R, et al. Order reduction for nonlinear dynamic models of distributed reacting systems. J Process Control. 2000;10:177-184.

[31] Kosambi D. Statistics in function space. J Indian Math Soc. 1943;7:76-88.

[32] Karhunen K. Uber Lineare Methoden in der Wahrscheinlichkeitsrechnung [Linear methods in probability]. Ann Acad Sci Fennicae Ser A1 Math Phys. 1946;37:3-79. 
[33] Loeve M. Fonctions Aléatoires du Second Ordre [Second order random functions]. In: Levy P, editor. Processus stochastiques et mouvement Brownien [Stochastic processes and Brownian motion]. Paris: Gauthier-Villars; 1948.

[34] Obukhov M-A. Statistical description of continuous fields. Tr Geophys Int Acad Nauk USSR. 1954;24:3-42.

[35] Pougachev V-S. General theory of the correlations of random functions. Izv Akad Nauk USSR. 1953;17:1401-1402.

[36] Watanabe S. Karhunen-Loève expansion and factor analysis theoretical remarks and applications. In: Proceedings of the 4th Conference on Information Theory, Prague, Czech Republic; Prague (NY): Academia Press; 1965.

[37] Mees A-I, Rapp P-E, Jennings L-S. Singular value decomposition and embedding dimension. Phys Rev A. 1987;36:340-346.

[38] Ravindra B. Comments on the physical interpretation of proper orthogonal modes in vibrations. J Sound Vib. 1999;219:189-192.

[39] Berthel B, Wattrisse B, Chrysochoos A, et al. Thermographic analysis of fatigue dissipation properties of steel sheets. Strain. 2007;43:273-279.

[40] Higham NJ. Matrix nearness problems and applications. In: Gover MJC and Barnett S, editors. Applications of matrix theory. Oxford: Clarendon Press, 1989; p. 1-27.

[41] Hubert L, Meuleman J, Heiser W. Two purposes for matrix factorization: a historical appraisal. SIAM Rev. 2000;42:68-82.

[42] Thomson W, Kelvin Lord. On the thermo-elastic and thermomagnetic properties of matter, Part I (dated 10th March, 1855). Q J Math. 1857:57-77. 